\title{
Modelagem e morfologia urbana com o PeopleGrid
}

\author{
Modeling and urban morphology with PeopleGrid
}

\author{
Mauricio Couto Polidori[a] (D), Otávio Martins Peres[a] (D), Fernanda Tomiello[b] (D)
}

[a] Universidade Federal de Pelotas (UFPel), Faculdade de Arquitetura e Urbanismo, Pelotas, RS, Brasil

[b] Universidade Católica de Pelotas (UCPel), Curso de Arquitetura e Urbanismo, Pelotas, RS, Brasil

Como citar: Polidori, M. C., Peres, O. M., \& Tomiello, F. (2020). Modelagem e morfologia urbana com o PeopleGrid. urbe. Revista Brasileira de Gestão Urbana, 12, e20190234. https://doi.org/10.1590/2175-3369.012.e20190234

\section{Resumo}

Modelagem urbana tem sido uma das maneiras para compreender a cidade, capturando parte da realidade e realizando experimentos, o que pode ser feito com sucesso em estudos em morfologia urbana. Com o uso crescente da internet, popularização e aumento da velocidade de transmissão de dados, modelos com operação on line representam uma alternativa interessante, o que pode trazer avanços na sua popularização, angariando participação e integrando com sistemas de informações geográficas. O objetivo deste artigo é apresentar a plataforma PeopleGrid, a qual consiste numa ferramenta para realizar perguntas e obter respostas sobre aspectos urbanos que têm como fundamento a localização, permitindo desenhar em mapas disponíveis na internet, sobre grids com células quadradas, com temas e níveis de desagregação espacial decididos pelos pesquisadores. Nos estudos de morfologia urbana, a plataforma oferece uma possibilidade de elaborar mapas mediante um processo colaborativo, os quais podem vir a representar desejos coletivos, obtidos pelo somatório das opiniões individuais. Desse modo, a plataforma PeopleGrid captura aspectos da realidade através do conhecimento parcial da cidade que cada pessoa possui, valorizando subjetividades e construindo uma nova informação, que pode ser reconhecida como uma intersubjetividade

Palavras-chave: Modelagem urbana. Morfologia urbana. Mapeamento colaborativo.

\begin{abstract}
Urban modeling is one of the ways to understand the city, capturing part of reality and conducting experiments, which can be done in studies on urban morphology. With the increasing use of the internet, popularization and increasing the speed of data transmission, models with online operation represent an interesting alternative, which can bring advances in its popularization, raising participation and integrating with geographic information systems. The purpose of this study is to introduce the PeopleGrid platform, which consists in a tool for asking questions and getting answers about urban aspects based on location, allowing drawing on maps available on the internet, about square cell grids with themes and spatial disaggregation levels decided by the researchers. In urban morphology studies, the platform offers
\end{abstract}

MCP é arquiteto e urbanista, doutor, e-mail: mauricio.polidori@gmail.com

FT é arquiteta e urbanista, mestre, e-mail: fernandatomiello@gmail.com 
a possibility of making maps through a collaborative process, which may represent collective desires, obtained by the sum of individual opinions. Thus, the PeopleGrid platform captures aspects of reality through partial knowledge of the city that each person owns, valuing subjectivities and building new information, which can be recognized as an intersubjectivity.

Keywords: Urban modeling. Urban morphology. Collaborative mapping.

\section{Introdução}

Estudar a morfologia urbana através de modelagem espacial tem sido um dos desafios da pesquisa contemporânea, para o que têm sido desenvolvidos diversos instrumentos auxiliares, dedicados à descrição e experimentação em tecidos urbanos, assim como simulação de possibilidades para o futuro das cidades. Nesse caminho, o grupo de trabalho do Laboratório de Urbanismo da Faculdade de Arquitetura da Universidade Federal de Pelotas (LabUrb - FAUrb - UFPel) vem trabalhando nas seguintes alternativas: a) modelagem intraurbana baseada em grafos, através do software UrbanMetrics, com representação do espaço urbano por pontos, linhas e áreas (UFPel, 2019a); b) simulação de crescimento urbano baseado em autômatos celulares, mediante o software CityCell, com representação do espaço urbano e seu entorno através de grids celulares retangulares (UFPel, 2019b); c) elaboração de questionários virtuais sobre mapas urbanos, o que é oferecido pela plataforma PeopleGrid (2019). É justamente para esse terceiro instrumento que o artigo está dedicado, apresentando relações com modelagem e morfologia urbana.

0 artigo apresenta seis partes, a saber: a primeira trata do que é a plataforma PeopleGrid, da ideia de trabalhar com células e das possibilidades de utilização; a segunda e a terceira partes apresentam uma aproximação com modelagem e morfologia urbana; a quarta mostra exemplos de aplicação, e a quinta e sexta tratam das conclusões e referências.

\section{O que é o PeopleGrid?}

PeopleGrid é uma plataforma na internet que permite uma possibilidade para opinar sobre a cidade, desenhando diretamente sobre mapas urbanos, disponíveis através de aplicativo computacional (PeopleGrid, 2019). Opera baseado em uma grade de células, através da qual é possível a captura e junção da opinião das pessoas, as quais informam no mapa seus conhecimentos ou desejos sobre o espaço. A abordagem é na linha dos mapas sociais urbanos e da modelagem urbana, com utilização de conceitos de Sistemas de Informações Geográficas (SIG) em processos participativos (PPSIG), como sugerem Buzai (2003), Batty (2003) e Bugs (2010).

A ideia de trabalhar com células está associada ao interesse na simplificação da elaboração de mapas, pois parece que marcar espaços discretos facilita os desenhos dos usuários, de modo diferente do que acontece com pontos, linhas e áreas, que exigem maior precisão e habilidade com o computador. De fato, os mapas sociais urbanos desenhados com vetores são frequentemente convertidos para grids para serem feitas análise espaciais, e no caso do PeopleGrid isso já é feito originalmente, em função de informações de lugares do tamanho mínimo de uma célula, que por hipótese já carregam considerações sobre as células vizinhas. É interessante considerar que os mapas produzidos pela plataforma podem participar de um conjunto mais amplo de mapas convencionais produzidos em SIG, permitindo comparações e novas análise espaciais.

Tecnicamente, o PeopleGrid é um SIG simplificado que disponibiliza um questionário virtual apoiado por um mapa dividido em uma grade de células ou grid, em que o valor de cada uma dessas células corresponde ao valor de uma variável que está sendo considerada no questionário (Campo, 1997). 0 mapeamento através deste método permite relacionar cada parcela do espaço com sua vizinhança, superando algumas limitações do pensamento e representações espaciais euclidianas (que utilizam 
pontos, linhas e áreas separadas) e avançando sobre possibilidades do espaço leibnitziano, de modo atento às relações de contexto (Polidori, 2004). Isso ocorre porque os usuários sempre informam localizações aproximadas e mediadas pelo tamanho das células, sendo a escolha e os limites fortemente influenciados pela vizinhança.

O PeopleGrid apresenta três possibilidades de utilização: como pesquisador, como respondente ou como observador. 0 pesquisador realiza a função de criar as questões de pesquisa, delimitar o local de trabalho e o nível de desagregação espacial, delineando o que será pesquisado. As questões de pesquisa exigem que a dimensão espacial das respostas seja fundamental, pois a lógica do trabalho é justamente a espacialização ou a captura da dimensão espacial de algum aspecto urbano, permitindo que uma representação colaborativa do espaço urbano que venha a ser construída possa ser utilizada em processos de análise espacial.

As questões implementadas na plataforma PeopleGrid estão sempre interessadas em identificar localizações para alguma condição ou função da cidade, podendo operar como levantamento, diagnóstico ou proposição. Como levantamento, têm sido recorrentes perguntas relativas à segurança e presença social, do tipo: "Quais as áreas mais inseguras para os pedestres?"; ou "Quais as áreas mais frequentadas por mulheres?". Esse tipo de pergunta normalmente pretende informações com dificuldade de serem alcançadas por técnicas tradicionais, que normalmente incluem relatos de espacialização difícil de ser realizada. No caso dos diagnósticos, a pergunta já inclui algum juízo de valor pelo respondente, como, por exemplo, nas questões vinculadas ao sistema cicloviário e arborização urbana: "Quais as áreas em que faltam estacionamentos para bicicletas (paraciclos)?"; ou “Onde a arborização nas calçadas é insuficiente para proteger os pedestres do calor?". Os resultados podem auxiliar na elaboração de projetos, normalmente articulados com outros mapas temáticos e com informações complementares sobre os lugares identificados. Por fim, as questões podem pretender propostas mais diretas feitas pelos respondentes, tendo sido frequentes as perguntas destinadas à expansão urbana e à preservação ambiental: "Quais as áreas mais adequadas para a expansão urbana?"; ou "Quais as áreas prioritárias para a criação de parques urbanos?". Na mesma linha têm sido recorrentes as perguntas diretas sobre a localização de equipamentos comunitários, como: “Onde colocar a nova rodoviária da cidade?" ou "Onde colocar o novo posto de saúde do bairro?". Alguns pesquisadores têm questionado sobre a possibilidade de apreender a opinião dos usuários sobre algum fato ou lugar urbano, porém não é essa a aplicação da plataforma, que é restritamente dedicada a questões cuja resposta é a localização, pois nada é verbalizado e tudo é demarcado no mapa.

Diversos locais de trabalho têm estimulado os pesquisadores que utilizaram o PeopleGrid até agora, como é o caso de Pelotas (RS), Porto Alegre (RS), Jaguarão (RS), Rio Branco (UY), Chuí (RS), Chuy (UY)e Vitória (ES), sendo que a plataforma permite acessar qualquer cidade do mundo que esteja mapeada no sistema do GoogleMaps ${ }^{\circledR}$, pois esse aplicativo está integrado no PeopleGrid. Os locais de trabalho podem ser visualizados através de mapas vetoriais, de imagens de satélite e por fotografias terrestres, pela integração com o sistema do StreetView ${ }^{\circledR}$.

Os locais podem ser investigados em diferentes níveis de desagregação espacial, conforme a extensão da área de interesse e as características do objeto de estudo a que se referem as perguntas. A desagregação espacial depende inicialmente do tamanho total do local de trabalho, pois quanto maior a área, maior será o tamanho de cada célula, em razão da limitação do aplicativo, que determina o tamanho mínimo da célula em função da escala de visualização do mapa, podendo ser estimada uma fração de mil do inverso da escala. Essa questão da desagregação implica a possibilidade de incluir ou não determinados componentes da paisagem no estudo, devendo ser ajustados a extensão da área e o tamanho da célula ao que é preciso visualizar nos mapas, como fator de escolha ou decisão pelo usuário. Sendo assim, uma célula de $200 \mathrm{~m}$, comum nos estudos realizados até agora, representará com dificuldade uma rua urbana, embora possa ser adequada para uma massa vegetada ou uma barragem, por exemplo. Todavia, reside justamente na capacidade de representação do sistema celular a possibilidade de abstrair o tamanho literal de alguma feição 
Como respondente, a participação se dá desenhando diretamente sobre o mapa, mediante acesso da plataforma na internet, não sendo revelada a identidade de quem respondeu. Cada resposta pode ser graduada em 3 níveis de intensidade, sendo o resultado a soma simples de todas as respostas, normalizada de zero a cem. Para graduar esses níveis de intensidade das respostas, estão utilizados os valores de 1, 3 e 9, a partir do proposto por Saaty (1980), sugerindo ao usuário diferenças marcantes entre cada escolha.

Como observador, basta acessar o site e verificar os resultados de cada pesquisa, selecionando a pergunta, o método de classificação e o número de classes. O programa oferece a classificação por extratos de valores das células, por quantidade de células e por intervalos naturais, conforme o interesse em visualizar o resultado priorizando os valores, os agrupamentos de células com alguma pontuação ou as células com maiores diferenças das demais, respectivamente. Existem ainda filtros para o perfil do respondente, nível escolar e renda familiar, o que permite visualização de múltiplos mapas temáticos. Para bem visualizar e entender essas possibilidades, é interessante acessar uma pesquisa em andamento, o que pode ser feito em PeopleGrid (2019).

\section{Modelagem urbana e PeopleGrid}

Modelagem urbana pode ser entendida como um modo de capturar algum aspecto da realidade e o replicar de maneira controlada, permitindo a realização de experimentos, apoiando trabalhos de pesquisa e auxiliando na tomada de decisões, incluindo aspectos teóricos, empíricos e metodológicos. Como a realização dos trabalhos de modelagem urbana normalmente ocorre com intenção de construir ou testar uma ideia, a aproximação com alguma teoria é evidente, o que aparece tanto na escolha ou produção de modelos, como no delineamento experimental. Do ponto de vista empírico, a modelagem se realiza ao escolher o local de trabalho e o modelo propriamente dito, sendo que ambos colaboram para determinar os limites e o escopo de assuntos passível de inclusão em cada caso. Com relação à metodologia, embora seja conhecida a aproximação entre a modelagem urbana e a pesquisa científica tradicional (com tema, problema, pergunta, hipótese, objeto de estudo, resultados esperados, testes de hipótese, observações e conclusões), temos sugerido que a modelagem urbana é também um exercício de criatividade, invenção e abstração.

A criatividade pode aparecer em dois momentos diferentes: na produção de modelos e no seu uso. A produção de modelos parece demandar capacidade de sintetizar e recriar a realidade, numa delicada união com modos de ver e interpretar o mundo. Ao usar algum modelo, aparece o desafio de associar o conhecimento do usuário com o problema que será modelado, decidindo as variáveis que o constituem e os resultados que podem ser encontrados. Nesse caminho, invenção aparece como resultado da formulação de novas perguntas e novas respostas, sugerindo possibilidades contraintuitivas e alcançando transgressões ao que é usualmente admitido. Para fazer isso, é preciso exercitar a abstração, quer pelo modo de representação que os modelos normalmente utilizam, quer pela interpretação dos resultados, ao mesmo tempo que se transcrevem dados abstratos para a realidade, dando uso aos modelos numa troca permanente entre abstração e realidade.

Diversos autores apontam para limites nas possibilidades de usos de modelos, como é o caso de suas possibilidades de predição (Batty \& Torrens, 2001). Todavia, temos indicado o uso de modelos não somente para a antecipação de cenários de futuro prováveis, mas também para a indicação de condições desejáveis para a cidade, sendo justamente esse um dos fundamentos da concepção do PeopleGrid. Além disso, a prática recente dos pesquisadores com o PeopleGrid tem apontado para o fortalecimento de diagnósticos espaciais urbanos, identificando lugares com concentração ou falta de algo na cidade (como é o caso de funções urbanas destinadas a serviços de saúde, educação, lazer, cultura, bancos, correios e correlatos), de ocorrência de áreas perigosas (como é o caso do trânsito e de lugares com assaltos recorrentes), de exclusão socioespacial (pela presença estratificada de gênero, etnia e grupos com comportamento preconceituoso), de proteção ambiental (apontando trechos de fragilidade ambiental e 
de maior interesse para a preservação), de dotação de parques, praças e de melhorias nas ruas das cidades, dentre inúmeras possibilidades que os usuários vêm propondo.

A ideia de modelagem contida no PeopleGrid está associada aos conceitos de não equilíbrio, complexidade, emergência e auto-organização. Um estado dominante de não equilíbrio não implica, todavia, desordem imponderável ou ausência de padrão: ao contrário disso, esses sistemas apresentam a propriedade de se auto-organizarem, comportando-se como sistemas complexos (Portugali, 2000), podendo a aparente desordem ser considerada como um modo superior de organização (Pena-Vega, 2003). Sistemas auto-organizáveis operam com independência de um núcleo central prescritor de regras, sendo que resultados emergem da dinâmica instaurada, podendo ser observados em seu processo evolutivo, sendo que no campo dos estudos da cidade e de sua dinâmica, esse conceito se aplica na elaboração de explicações para a produção do espaço e para as morfologias resultantes. Sistemas complexos normalmente apresentam a propriedade de auto-organização, sendo que as chamadas teorias ou ciências da complexidade aplicadas à cidade (O'Sullivan et al., 2001) assumem que os estados de um sistema urbano não são nem determinísticos, como os previstos nas teorias causais tradicionais, nem aleatórios, isto é, absolutamente imprevisíveis (Lucas, 2000). Essas ideias desafiam modelos e modelistas urbanos a representarem o espaço de modo mais próximo de sua realidade (Batty, 1998), e é isso que está sendo buscado aqui. Ao cabo, pode ser considerado que os mapas coletivos produzidos com o PeopleGrid emergem de múltiplas colaborações on line na internet, sendo esperado que padrões morfológicos possam ser reconhecidos e acumulados a cada novo experimento.

A estrutura do projeto PeopleGrid foi definida com a utilização do framework CodeIgniter como base para a programação, sendo que no espaço cartográfico é utilizado o Google Maps para suprir a necessidade de manipulação de mapas, além do banco de dados PostgreSQL, para realizar a persistência dos dados (Presmann, 1995; Gabardo, 2010).

0 input é realizado de duas maneiras, conforme o seu papel em cada pesquisa, se como pesquisador ou como respondente. Como pesquisador, o input é realizado através da escolha do local de trabalho, de seus limites e da desagregação espacial, definindo o grid de trabalho. 0 pesquisador também é responsável pela elaboração das perguntas que vão determinar o assunto de interesse e orientar as pessoas que forem participar com respostas. Como respondentes, aparece uma espécie de segunda camada de input, elaborada pelas pessoas individualmente, ao acessar a plataforma no computador. Se, por um lado, essas respostas são resultado ao que questionou o pesquisador, por outro, são parte do input que constitui a resposta coletiva, a qual é uma superposição de todas as respostas individuais.

A legitimação dos resultados pode ser feita através de mapas analógicos com as mesmas questões utilizadas na internet, com quantidade e perfil de respondentes limitados, mediante o grau de similaridade dos resultados. Também é importante considerar as possibilidades de consulta a informantes qualificados, os quais podem discutir os mapas gerados no PeopleGrid em relação a resultados esperados. Ainda pode ser valioso investigar a aproximação dos resultados com o alcançado em trabalhos anteriores, construindo séries e identificando as suas diferenças. Na prática, tem sido interessante observar alguma estabilidade nos resultados, apesar do aumento no número de respostas, o que sugere consistência na modelagem e pode implicar validação da pesquisa. Além disso, é possível pensar em aproveitar os mapeamentos obtidos com o PeopleGrid como referência para legitimação de experimentos realizados com outros modelos, postas suas bases empíricas e fundadas nas informações oferecidas pelas pessoas.

\section{Morfologia urbana e PeopleGrid}

Morfologia urbana é o estudo dedicado da forma das cidades, com notável independência disciplinar, relacionando a forma urbana com o processo social (Hillier \& Hanson, 1984; Lamas, 1993; Oliveira, 2018) e com o ambiente natural (Pesci, 2000; Alberti et al., 2003; Polidori, 2004), o que está denominado aqui de processo socioambiental. Ao realizar essas relações entre forma urbana, sociedade e natureza, três 
maneiras têm sido recorrentes: a) a forma da cidade dependeria do processo socioambiental, dele derivando; desse modo, para entender a forma urbana seria necessário entender antes a sociedade e a natureza, as quais conteriam os ditames teóricos e físicos para a compreensão da forma urbana; b) de modo oposto, outra maneira de compreender sugere que a forma urbana implicaria de modo decisivo no processo socioambiental, condicionando fortemente o comportamento humano e as organizações socias, inclusive reformatando a natureza através do desenho da cidade; desse modo, os arranjos espaciais, a estrutura urbana e as funcionalidades nela contidas assumiriam papel de protagonismo, com forte independência dos processos socioambientais; c) uma terceira maneira aponta para a inseparabilidade entre forma urbana e questões socioambientais, não havendo hegemonia de uns sobre os outros; sendo assim, uma mudança da forma urbana implicaria inexoravelmente noutra no processo socioambiental e vice-versa, como se houvesse uma forte e mútua relação de causa e efeito envolvida.

Diferentemente dessas três possibilidades, temos defendido que a forma urbana nem depende, nem determina, nem é inseparável do processo socioambiental, indicando que forma urbana de fato é uma das dimensões da cidade, a qual tem diversas outras dimensões, que aparecem em relações complexas, raramente completas e com possibilidades explicativas parciais sobre as cidades. Nesse modo de pensar, a forma urbana é compreendida como uma dimensão fundamental, sem prejuízo de outras dimensões, como a social, natural, cultural, política, econômica, funcional e temporal, sendo que cada uma dessas dimensões apresenta possibilidades explicativas dentro do seu escopo, cuja completude pode ser maior ou menor, conforme o desenvolvimento de cada área disciplinar, o esforço de campo aplicado em cada caso e de acordo com cada cidade, que pode ter uma ou outra dimensão mais potente que as demais. Nesse contexto, a dimensão morfológica da cidade parece ser fundamental, sendo camada inicial para seu entendimento, com decidida independência disciplinar e específico suporte teórico.

Considerando que o resultado dos trabalhos com o PeopleGrid é sempre através de grids, com células e classificações, definido agrupamentos em classes, a morfologia urbana pode ser representada através de manchas (Forman, 1995). Possibilidades de estudar a morfologia de manchas aparece em ecologia de paisagem, onde diversos autores convergem para representar a paisagem por corredores, manchas e matrizes (Pascual et al., 2011). No caso das manchas, alguns descritores podem qualificar e até quantificar suas características, apontando formas, tamanhos, bordas, diversidade e densidade (McGarigal \& Marks, 1994), onde assumem importância a quantidade de células em cada categoria, a continuidade espacial, a fragmentação de cada agrupamento, a linearidade e a compacidade de cada classe (a utilização dessas medidas está aplicada para o caso urbano no trabalho de Toralles, 2012).

Mesmo frente às possibilidades dessas medidas de manchas, a principal contribuição do PeopleGrid para a morfologia urbana parece estar na elaboração de mapas através da opinião das pessoas, construindo um modo diferente do derivado diretamente das feições do território, dos espaços abertos e construídos, manifesto em células e seus agrupamentos, os quais são determinados pelo somatório de respostas individuais. Uma vez que as respostas são desenhos realizados diretamente no grid, versões da cidade imaginadas ou desejadas pelas pessoas são permitidas pela ferramenta, o que pode ajudar em estudos urbanos.

\section{Exemplos de aplicações do PeopleGrid}

O PeopleGrid apresenta abrangente possibilidade de escolha do lugar a utilizar, pois opera sobre a plataforma on line do GoogleMaps, a qual inclui qualquer lugar do mundo, representado por trechos urbanos ou rurais, encontrados por ferramentas de busca de cidade por nome ou por coordenadas geográficas. Embora os recortes espaciais sejam sempre retangulares, definidos pelo formato da tela dos computadores e do grid, que trabalha sempre com células quadradas, não há limitações sobre a proporção da área de trabalho, que pode assumir formato com predominância vertical ou horizontal, oportunizando o estudo em locais com diferentes morfologias na área de interesse. 
A utilização do PeopleGrid pode interessar em diferentes etapas do processo tradicional de planejamento urbano, incluindo levantamento, diagnóstico, prognóstico, conceituação e elaboração de propostas. Pesquisas já realizadas indicam possibilidades para utilização da plataforma PeopleGrid no planejamento urbano e ambiental, como são os casos das cidades do Chuí (RS), Chuy (UY) e Pelotas, apresentados adiante. No caso das cidades do Chuí (RS) e Chuy (UY), na fronteira entre o Brasil e Uruguai, o grid utilizado foi de $100 \mathrm{~m}$, num total de 2.800 células, cada uma com área de 1 ha. 0 local de trabalho, as perguntas e as respostas obtidas aparecem nas Figuras 1, 2, 3 e 4. Foram feitas as seguintes perguntas: Quais áreas destinar para expansão urbana? Quais áreas destinar para preservação ambiental? Quais áreas destinar para parque urbano público?

No caso da cidade de Pelotas (RS), o grid utilizado foi de $200 \mathrm{~m}$, num total de 18.000 células, cada uma com área de 4ha. 0 local de trabalho, as perguntas e as respostas obtidas aparecem nas Figuras 5 e 6. Foi feita a pergunta: Quais as áreas a destinar para parque na área urbana?

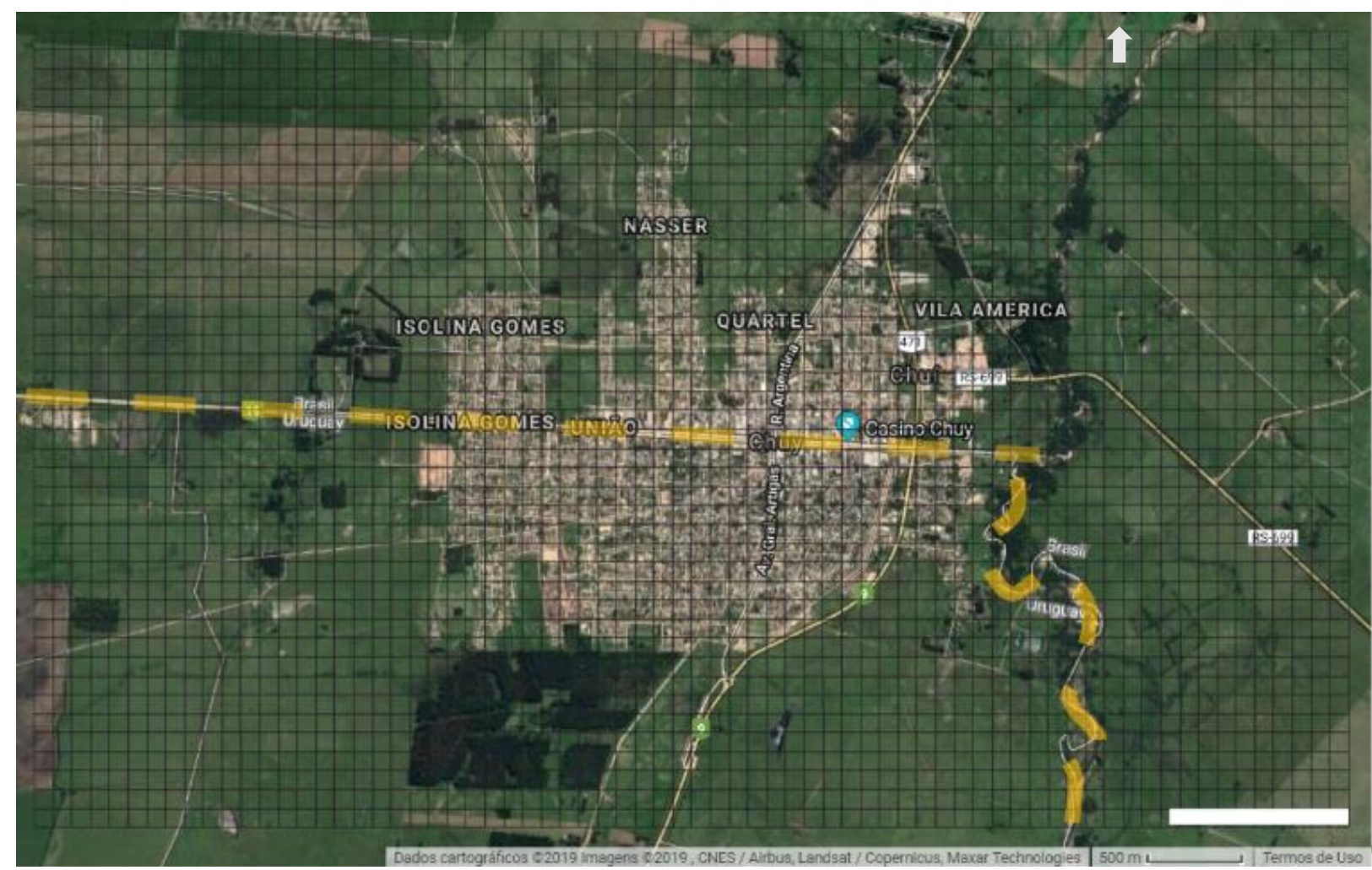

Figura 1 - Imagem de satélite mostrando o local de trabalho, com as cidades do Chuí (RS) acima e Chuy (UY) abaixo da linha tracejada amarela; em preto aparece o grid de 2.800 células de 100m. Fonte na plataforma do PeopleGrid (2019). Dos autores (2019). 


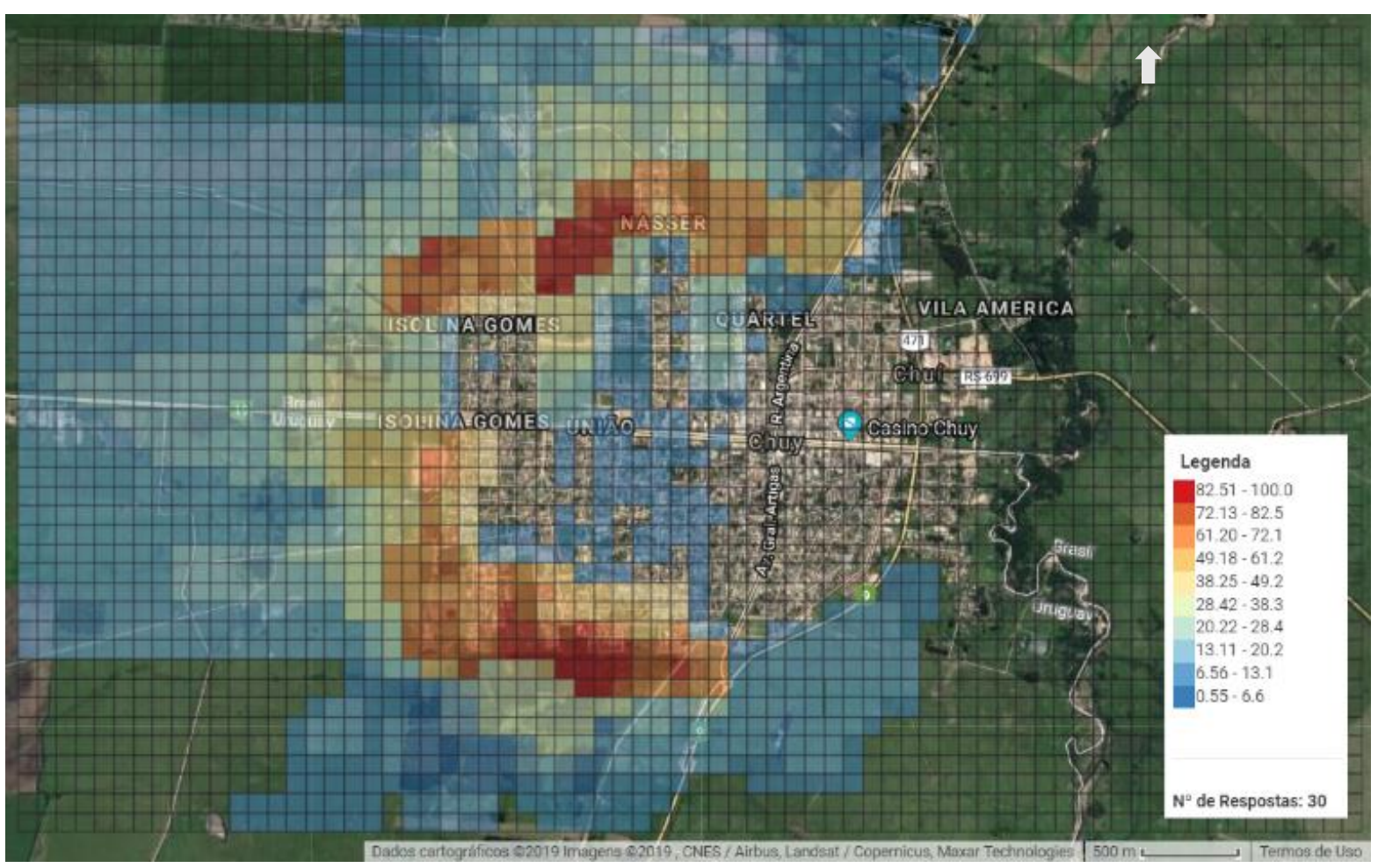

Figura 2 - Resultado da pergunta "Quais áreas destinar para a expansão urbana?", com visualização normalizada de 0 a 100, em 10 classes, por natural breaks, usando vermelho para os valores mais elevados. Fonte na plataforma do PeopleGrid (2019). Dos autores (2019).

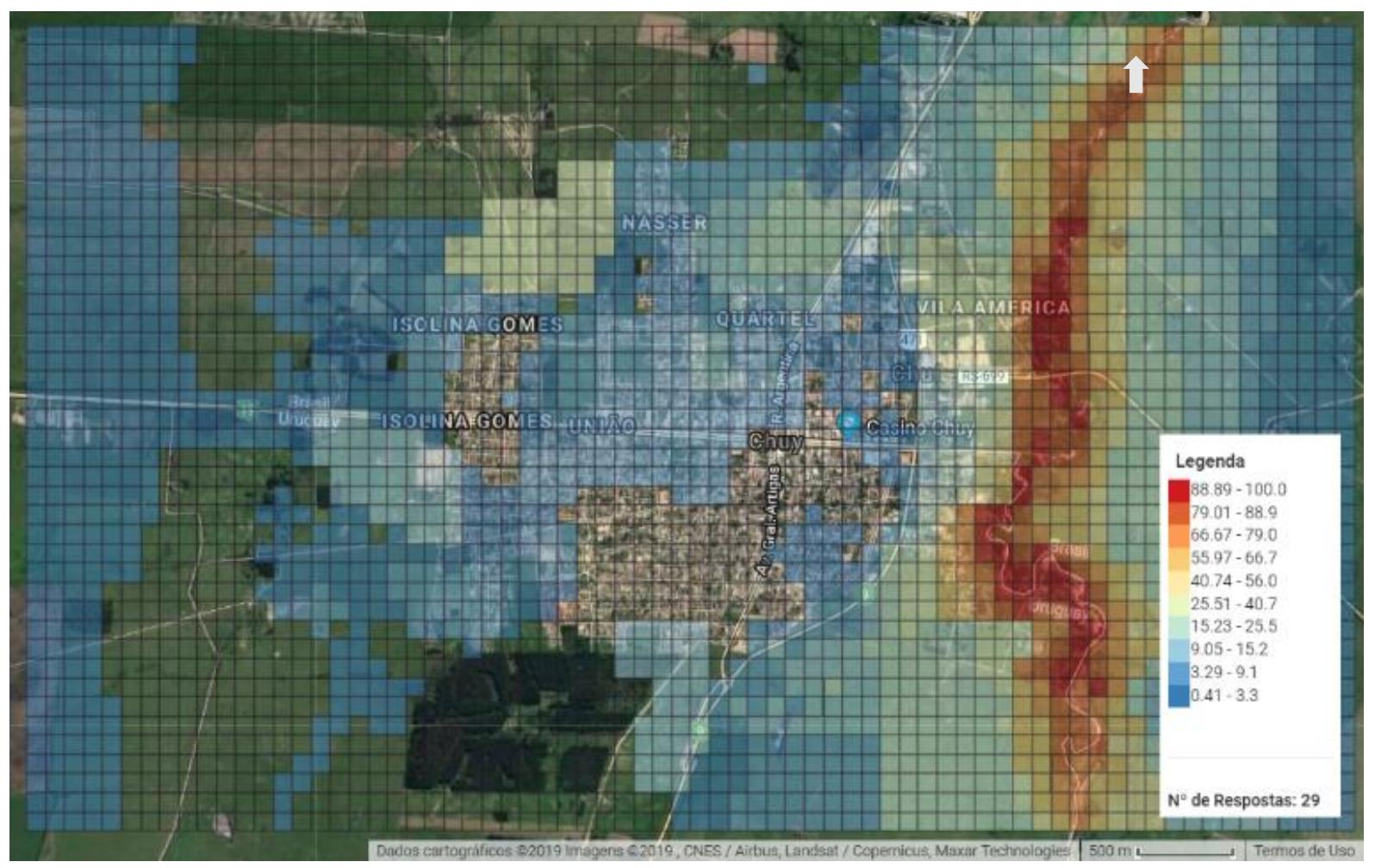

Figura 3 - Resultado da pergunta "Quais áreas destinar para a preservação ambiental?", com visualização normalizada de 0 a 100, em 10 classes, por natural breaks, usando vermelho para os valores mais elevados. Fonte na plataforma do PeopleGrid (2019). Dos autores (2019). 


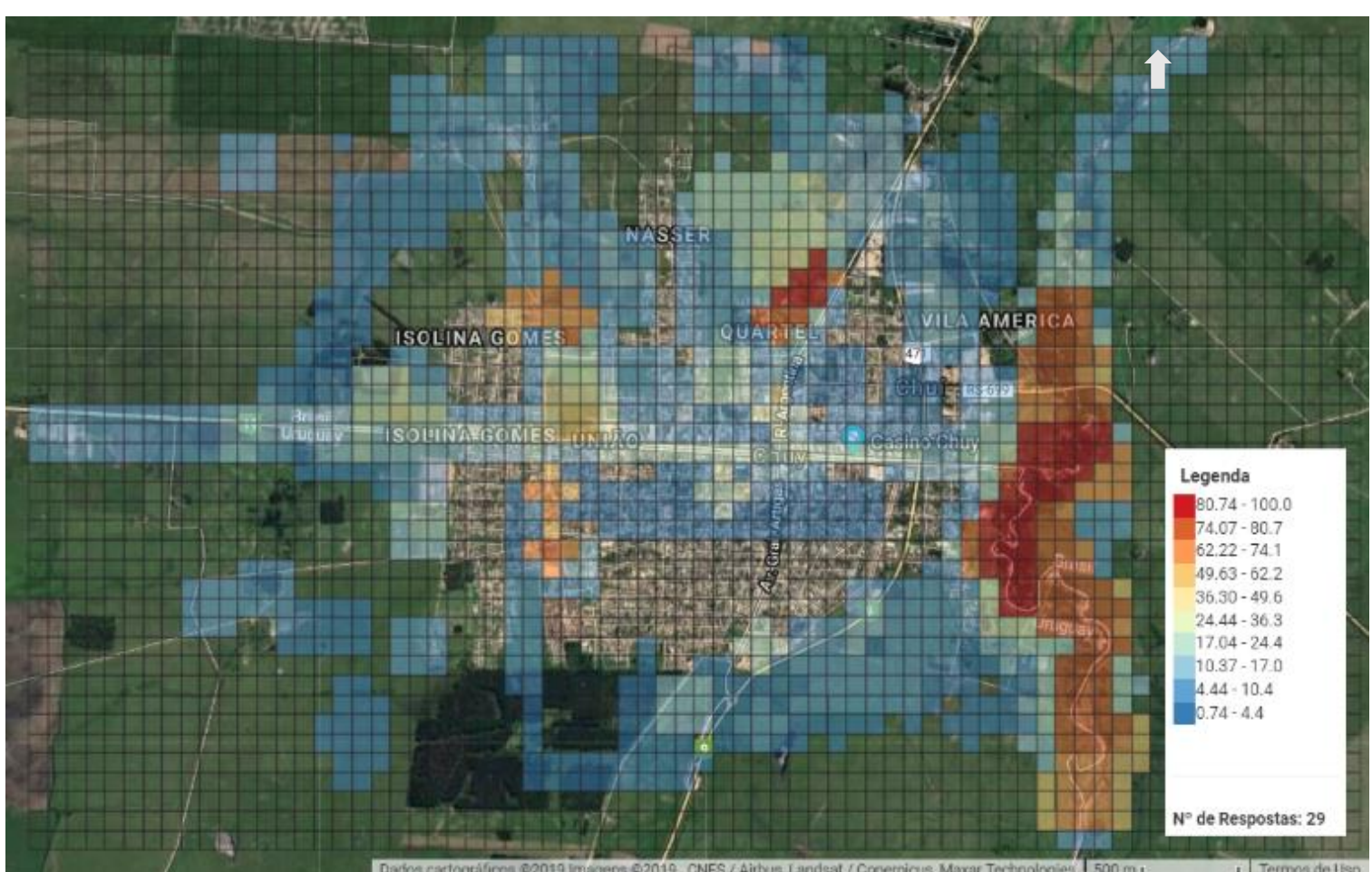

Figura 4 - Resultado da pergunta "Quais áreas destinar para parque urbano público?", com visualização normalizada de 0 a 100, em 10 classes, por natural breaks, usando vermelho para os valores mais elevados. Fonte na plataforma do PeopleGrid (2019). Dos autores (2019).

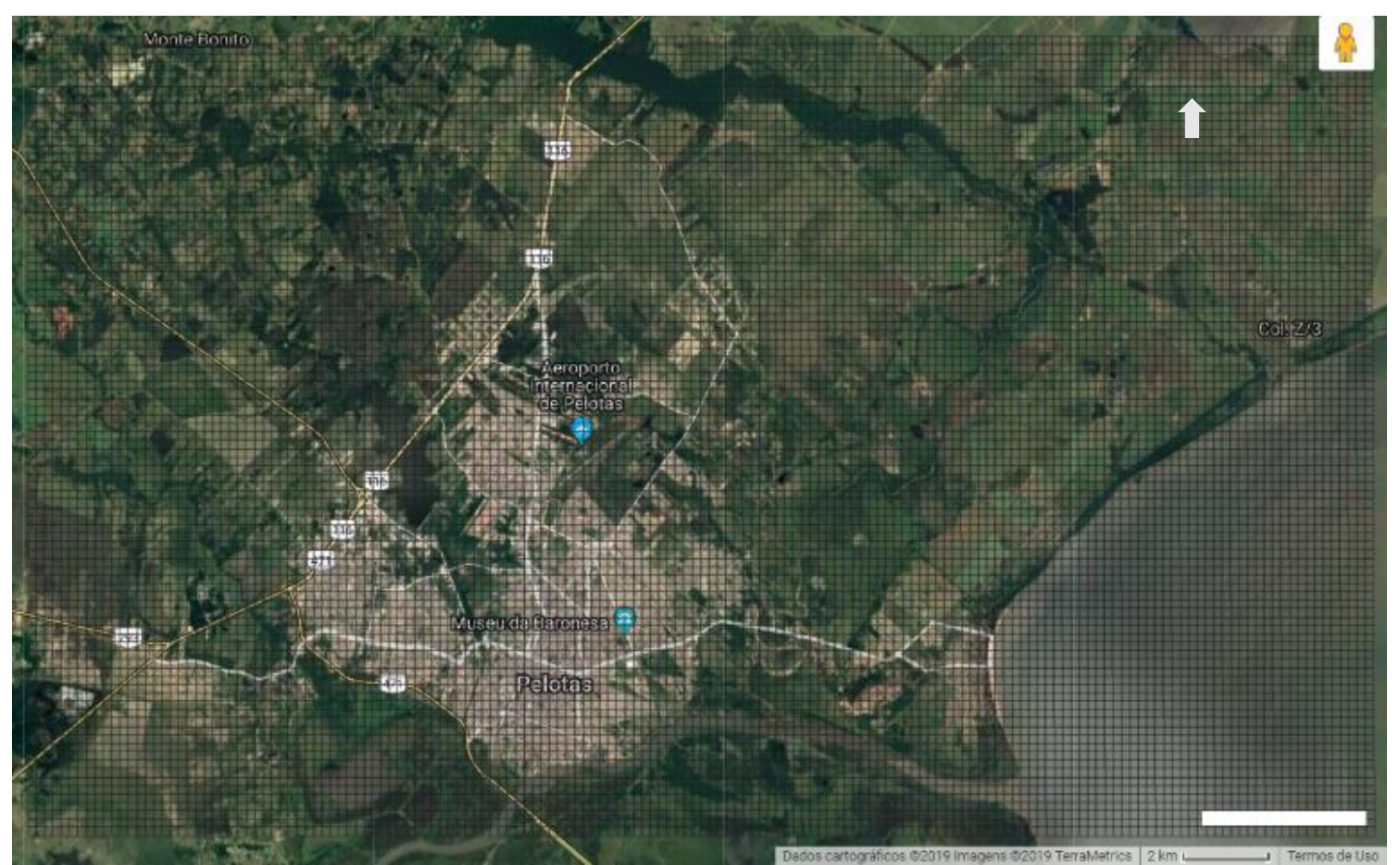

Figura 5 - Imagem de satélite mostrando o local de trabalho em Pelotas (RS); em preto aparece o grid de 18.000 células de 200m. Fonte na plataforma do PeopleGrid (2019). Dos autores (2019). 


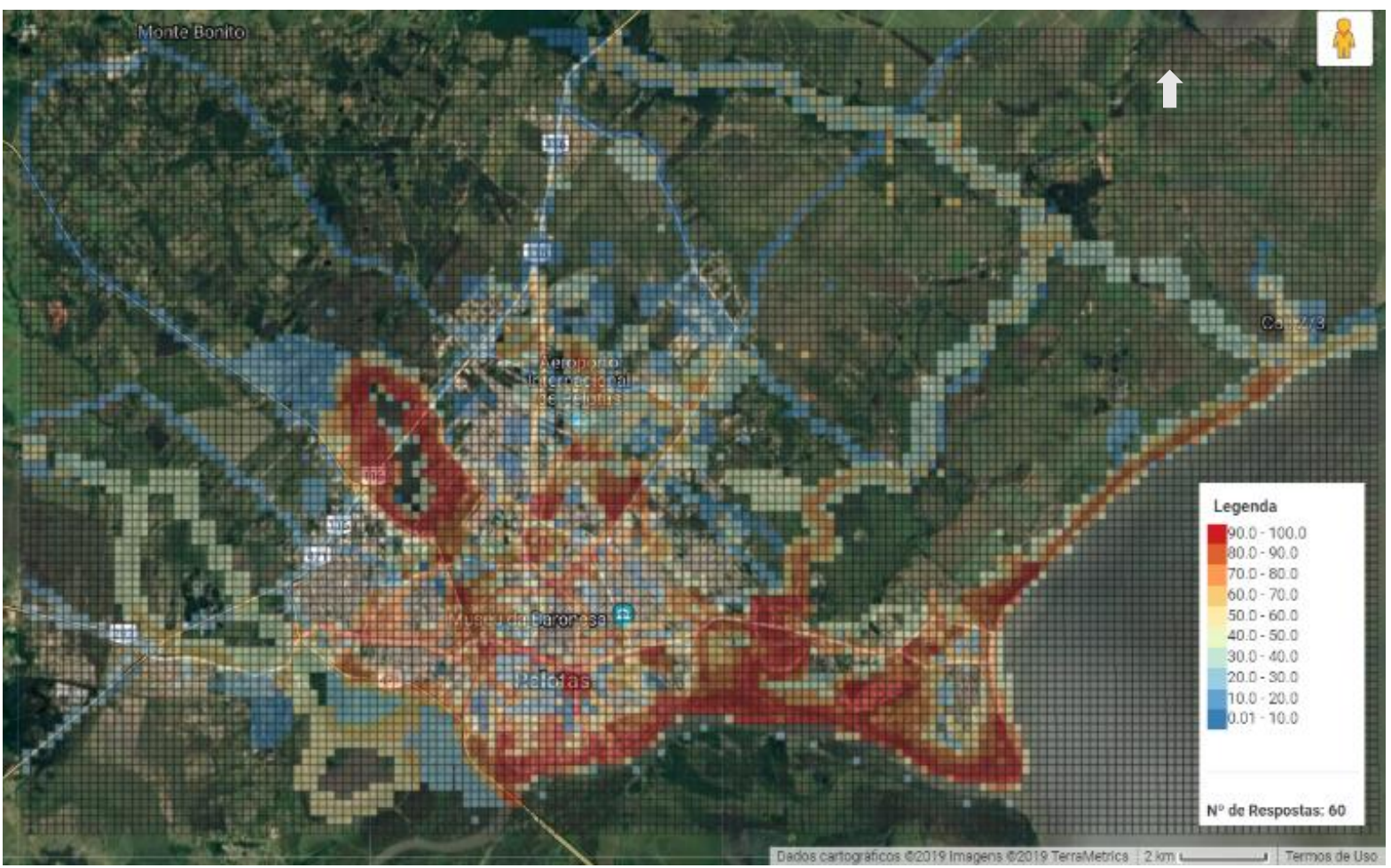

Figura 6 - Resultado da pergunta "Quais as áreas a destinar para parque na área urbana?", com visualização normalizada de 0 a 100, em 10 classes, por natural breaks, usando vermelho para os valores mais elevados.

Fonte na plataforma do PeopleGrid (2019). Dos autores (2019).

No caso das cidades de fronteira entre o Brasil e o Uruguai, Chuí (RS) e Chuy (UY), foi possível observar que as respostas sugerem expansões urbanas simétricas nas bordas sul e norte das duas cidades, com as áreas de preservação principais no entorno do Rio Chuí. Também pode ser observada a sugestão de criação de um parque urbano coincidente com parte da área de preservação ambiental, o que implica projeto que resolva as vantagens e conflitos dessa superposição. Esse resultado traz interessante contribuição para o planejamento urbano das duas cidades, pois aponta possibilidade de crescimento minimizando conflitos com a preservação ambiental.

No caso de Pelotas (RS), o mapeamento está demarcando como prioridade para parque urbano as áreas próximas de recurso hídricos, destacando o Canal de São Gonçalo (abaixo), o Arroio Pelotas e a Laguna dos Patos (à direita) e a Barragem do Arroio Santa Bárbara (à esquerda). Esse resultado sugere a integração das funções urbanas com as águas próximas da cidade, as quais apresentam grande potencial para o sistema de áreas abertas, sendo seu aproveitamento como parques de elevada importância para a qualidade de vida natural na cidade.

Uma limitação que está agendada para ser enfrentada é a integração com outras plataformas com informações disponíveis na internet, como OpenStreetMap e MapBiomas, de modo que o respondente possa buscar informações sobre o local da pesquisa. Interessa também desenvolver possibilidades de visualização de mapas do IBGE, particularmente de demografia e densidades populacionais, o que está pautado para as próximas versões da plataforma do PeopleGrid.

\section{Conclusões}

A ideia de produzir mapas de modo participativo tem evoluído junto com o desenvolvimento de novas tecnologias, o que pode incluir os SIGs e a internet. Embora o uso dos SIGs normalmente esteja limitado a especialistas em geoprocessamento e planejamento urbano, assim como os trabalhos com cartografia social frequentemente estão distantes do uso de recursos digitais, a produção de mapas coletivos pela 
internet parece trazer esperança na elaboração de produtos participativos com maior qualidade e possibilidade de utilização. Esses mapas podem vir a representar desejos coletivos, obtidos pelo somatório das opiniões individuais. Nesse caminho, pode ser considerado que a plataforma PeopleGrid captura aspectos da realidade através do conhecimento parcial da cidade que cada pessoa possui, valorizando subjetividades e construindo uma nova informação, a qual pode ser reconhecida como uma intersubjetividade. Essa proposta pode ser considerada inovadora e operar como ferramenta na área de planejamento urbano, proporcionando a pesquisadores, gestores e especialistas um modo de incluir pessoas no processo de tomada de decisão. Além disso, pode disponibilizar ao pesquisador flexibilidade na construção da consulta à população, podendo ser aplicado em inúmeras cidades.

Um diferencial da ferramenta é a facilidade de compreensão por parte do usuário, que pode se situar entre leigo e especialista no assunto de planejamento urbano. Sendo assim, a expectativa é de poder alcançar diversos tipos de indivíduos, incluindo suas cognições, juízos de valores e culturas sobre o meio urbano, aspectos que parecem ser capturados com alguma dificuldade nos sistemas de informações geográficas e nos instrumentos de análise espacial convencionais.

No campo da morfologia urbana está a ideia de que as descrições que as pessoas fazem das cidades, incluindo suas interpretações e desejos, podem constituir mais uma camada para a compreensão da forma urbana, de modo interativo e carregando aspectos de complexidade, auto-organização e emergência. Nesse caminho, a ideia é produzir um mapa colaborativo que possa ser incluído em outros processos de análise espacial urbana, constituindo uma camada a mais, com informações sobre desejos ou necessidades das pessoas.

Ao cabo, é importante considerar que as possibilidades criadas por novos (e velhos) instrumentos de apoio ou de suporte à decisão não substituem os embates e as lutas políticas dos movimentos urbanos, sociais e ambientais. Pelo contrário, esses instrumentos podem, como é o caso do PeopleGrid, fortalecer as pautas e a autonomia populares, fundamentais para a transformação das cidades e para o encontro de alternativas para o futuro. A intenção dos autores é de priorizar as pesquisas dedicadas à preservação ambiental, qualidade de vida e equidade espacial urbana, em detrimento de experimentos que possam concorrer para acirrar a concentração de riquezas, a supremacia do capital sobre o trabalho e a segregação socioespacial. Ao mesmo tempo que os estudos de morfologia e modelagem urbana demandam autonomia disciplinar, os movimentos socioambientais requerem qualidade de vida e valorização dos interesses coletivos, justa seara da proposta da plataforma PeopleGrid.

\section{Referências}

Alberti, M., Marzluff, J. M., Shulenberger, E., Bradley, G., Ryan, C., \& Zumbrunnen, C. (2003). Integrating humans into ecology: opportunities and challenges for studying urban ecosystems. Bioscience, 53(12), 1169-1179.

http://dx.doi.org/10.1641/0006-3568(2003)053[1169:IHIEOA]2.0.CO;2.

Batty, M. (1998). Urban evolution on the desktop: simulation with the use of extended cellular automata. Environment \& Planning A, 30(11), 1943-1967. http://dx.doi.org/10.1068/a301943.

Batty, M. (2003). Experiments is Web-based PPGIS: multimedia in urban regeneration. In P. A. Longley, \& M. Batty (Eds.), Advanced spatial analysis (Chap. 8). California: ESRI.

Batty, M., \& Torrens, P. (2001). Modeling complexity: the limits to prediction. London: Casa, UCL. Recuperado em 10 de julho de 2019, de https://www.ucl.ac.uk/bartlett/casa/sites/bartlett/files/migrated-files/paper36_0.pdf

Bugs, G. (2010). Uso da cartografia digital interativa para a participação popular na gestão e planejamento urbano (Dissertação de mestrado). Programa de Pós-graduação, Universidade Federal do Rio Grande do Sul, Porto Alegre. Recuperado em 10 de julho de 2019, de https://www.lume.ufrgs.br/bitstream/handle/10183/112006/000953046.pdf

Buzai, G. D. (2003). Mapas sociales urbanos. Buenos Aires: Lugar.

Campo, M. R. (1997). Compreensão visual de frameworks através da introspeção de exemplos (Tese de doutorado). Programa de Pós-graduação em Ciência da Computação, Universidade Federal do Rio Grande do Sul, Porto Alegre. Recuperado em 10 de julho de 2009, de https://lume.ufrgs.br/handle/10183/17972 
Forman, R. T. T. (1995). Land mosaics: the ecology of landscapes and regions. New York: Cambridge University Press. http://dx.doi.org/10.1017/9781107050327.

Gabardo, A. C. (2010). CodeIgniter framework PHP. São Paulo: Novatec.

Hillier, B., \& Hanson, J. (1984). The social logic of space. Cambridge: Cambridge University Press. http://dx.doi.org/10.1017/CB09780511597237.

Lamas, J. M. (1993). Morfologia urbana e desenho da cidade. Lisboa: Dinalivro.

Lucas, C. (2000). Complexity and artificial life: what are they? Manchester: CALResCo.Group. Recuperado em 10 de julho de 2019, de http://calresco.org/cal.htm

McGarigal, K., \& Marks, B. J. (1994). Fragstats: spatial pattern analysis program for quantifying landscape structure, version 2.0. Amherst, MA: University of Massachusetts. Recuperado em 10 de julho de 2019, de http://www.umass.edu/landeco/pubs/mcgarigal.marks.1995.pdf

O'Sullivan, D., Martin, D., \& Ioannis, C. (2001). Complex spatial systems: the modelling foundations of urban and regional analysis. Environment and Planning. B, Planning \& Design, 28(3), 475-478.

http://dx.doi.org/10.1068/b2803rvw.

Oliveira, V. (Ed.). (2018). Diferentes abordagens em morfologia urbana: contributos luso-brasileiros. Porto: FEUP. Recuperado em 10 de julho de 2019, de https://vitoroliveira.fe.up.pt/pdf/diferentes-abordagens-em-morfologiaurbana.pdf

Pascual, V., Aguilera, F., Gómez, M., Barreira, P., Santos, J. M., \& Sendra, J. B. (2011). Métodos de comparación de mapas simulados del crecimiento urbano con los mapas reales. In XII CONFIBSIG - Conferencia Iberoamericana en Sistemas de Información Geográfica (pp. 47-65). Toluca, México: Instituto Literario, Universidad Autónoma del Estado de México. Recuperado em 10 de julho de 2019, de

https://www.academia.edu/7519011/Libro_CONFIBSIG

Pena-Vega, A. (2003). O despertar ecológico: Edgar Morin e a ecologia complexa (R. Nascimento \& E. Nascimento, trad.). Rio de Janeiro: Garamond.

PeopleGrid. (2019). Recuperado em 10 de julho de 2019, de http://www.peoplegrid.com.br/

Pesci, R. (2000). Del Titanic al Velero. La Plata: Cepa.

Polidori, M. (2004). Crescimento urbano e ambiente: um estudo exploratório sobre as transformações e o futuro da cidade (Tese de doutorado). Programa de Pós-graduação em Ecologia, Universidade Federal do Rio Grande do Sul, Porto Alegre. Recuperado em 10 de julho de 2019, de http://hdl.handle.net/10183/6191

Portugali, J. (2000). Self-organization and the city. Berlin: Springer. http://dx.doi.org/10.1007/978-3-662-04099-7.

Presmann, R. S. (1995). Engenharia de software: uma abordagem profissional (8. ed.). São Paulo: AMGH.

Saaty, T. L. (1980). The analytic hierarchy process. New York: McGraw-Hill.

Toralles, C. P. (2012). Cidade e crescimento periférico: modelagem e simulação da formação de periferias urbanas com autômatos celulares (Dissertação de mestrado). Programa de Pós-graduação em Arquitetura e Urbanismo, Faculdade de Arquitetura e Urbanismo, Universidade Federal de Pelotas, Pelotas. Recuperado em 10 de julho de 2019, de https://wp.ufpel.edu.br/prograu/dissertacoes-urbanismo-contemporaneo/2/

Universidade Federal de Pelotas - UFPel. (2019a). UrbanMetrics. Pelotas. Recuperado em 10 de julho de 2019, de https://wp.ufpel.edu.br/urbanmetrics/

Universidade Federal de Pelotas - UFPel. (2019b). CityCell. Pelotas. Recuperado em 10 de julho de 2019, de https://wp.ufpel.edu.br/citycell/

Editores convidados: Vitor Oliveira (Universidade do Porto, Portugal) e Bruno Zaitter (PUCPR, Brasil)

Recebido: Out. 25, 2019

Aprovado: Abr. 14, 2020 